\title{
Abd al Malik: le fluide vital du rap
}

\section{Tello-Collado, Santiago}

Universitat de València, santiago.tello@uv.es

\begin{abstract}
Resumen
Uno de los aspectos más llamativos, al tiempo que complejos de la música rap es lo que conocemos como flow. Partiendo de su sentido más orgánico, en tanto que líquido en movimiento, el flow en el rap requiere estar provisto de una enorme y cristalina vitalidad en el recitado de los versos, lo que le confiere uno de los aspectos más atractivos al tiempo que complejos en este tipo de música. Procedente de la locución americana to rap, es decir, entre chismorrear o darle al pico o, simplemente contar cualquier cosa, el rap song puede definirse como la dicción medio cantada y medio hablada de textos elaborados, rimados y ritmados sobre una base musical de mezclas de extractos de otras fuentes sonoras denominadas samples. Esta manifestación como tal, en tanto que una determinada performance que se lleva a cabo en la calle, debe entenderse como surgida en los 70 en Nueva York (Lapassade et Rousselot, 1998). El rap debe su exégesis a la música popular jamaicana, siendo el género demandado en los 70 entre la militancia negra estadounidense. Ya casi en los 80, observamos el resultado de esta música capaz de mezclar baile y política. No olvidemos pues que, al igual que el jazz, el blues, el soul o el rhythm and blues, el rap es parte integrante del arte negro americano. A diferencia de lo que pasó en los EEUU, el rap francés no comienza en la calle sino que estaba dirigido o bien a los media o al show. Abd Al Malik, imprime a su rap un cristalino y personal tratamiento con su particular flow.
\end{abstract}

Palabras clave: rap; flow; poesia; Abd al Malik.

\section{Résumé}

L'un des aspects les plus caractéristiques, ainsi que complexes, de la musique «rap» est ce que nous connaissons comme «flow». En partant de son sens le plus organique, en tant que liquide en mouvement, le «flow» dans le «rap» doit être pourvu d'une grande vitalité «cristalline» dans le récit des vers, ce qui lui attribue l'une des apparences les plus attirantes, et difficiles en même temps, dans ce type de musique. Le verbe «to rap» veut dire en argot américain «bavarder», «critiquer» ou tout simplement «parler» de n'importe quel sujet. Ainsi la «rap song» pourrait être définie comme la diction, moitié chantée, moitié parlée, de textes élaborés, rimés et rythmés sur une base musicale de mélanges d'extraits d'autres sources sonores nommées «samples». Cette manifestation, considérée comme un type de «performance» ayant lieu dans la rue, doit être située et comprise dans le contexte new-yorkais des années 1970 (Lapassade et Rousselot: 1998). En effet, le «rap» doit être expliqué et interprété en rapport avec la musique populaire jamaïcaine, sans aucun doute le genre musical le plus demandé dans les années 1970 dans le milieu du militantisme noir américain. Au tournant des années 1980, on constate clairement les résultats de cette musique qui mélange la danse et la politique. Il ne faudrait donc pas oublier que le «rap», tout comme le «jazz», le «blues», la «soul» ou le «rhythm and blues», occupe une place importante dans l'expression artistique afro-américaine. Contrairement à ce qui s'est passé aux États-Unis, le «rap» français ne commence pas dans la rue, il est plutôt dirigé vers les médias ou les spectacles. Dans ce contexte, Abd al Malik, imprime à son «rap» une marque personnelle et «cristalline» avec son «flow» particulier.

Mots-clés: rap; flow; poésie; Abd al Mali.

\footnotetext{
Abstract

One of the aspects, more complex and outstanding of the music rap is what we know as flow. From its most organic sense, as well as liquid in movement, the flow in the rap needs to be provided with an enormous and crystalline vitality in the recitation of the verses, which gives to it one of the most attractive aspects at
} 
the same time as complexity in this type of music. Proceeding from the American expression 'rap', that is to say, between gossiping or chatting or, simply telling anything, the rap song can be defined as the diction half way spoken with elaborated, rhymed texts on a musical base of mixtures of extracts of other sonorous sources called samples. This manifestation as it is, that is, meaning a certain performance that is carried out in the street, must be understood like arisen in the 70s in New York (Lapassade and Rousselot : 1998).The rap owes his exegesis to the popular Jamaican music, being the musical style demanded in the 70 s among the black American militancy. Almost in the 80s, we observe the result of this kind of music capable of mixing dance and politics. Let's not to forget that, as the jazz, the blues, the soul or the rhythm and blues, the rap is an integral part of the black American art. Unlike what happened in the USA, the French rap does not begin in the street. It was directed to the media or to the show. Abd al Al Malik, stamps to his rap crystalline and personal treatment with his individual flow.

Keywords: rap; flow; poetry; Abd al Malik.

\section{Introducción}

La elección de Abd al Malik, músico, que aglutina facetas como las de rapero, eslamero, poeta, escritor y realizador ha venido determinada por la relevancia y particular fluidez de su flow, sin el que la dicción, declamación o escansión en el rap tendría sentido. Se suele afirmar que una de las características más destacadas y distintivas del rap es la enorme maestría y extrema fluidez con las que el rapero declama un texto partiendo de una base musical. Hemos creído, por tanto, realizar un acercamiento a la particular utilización del flow de Abd al Malik, el cual hemos calificado como un elemento vital como el agua. ¿Por qué? Diferentes estudiosos como Christian Béthune (2003: 235) o Isabelle Marc (2007: 6), en sendas aportaciones sobre la estética del rap, hacen referencia a este concepto difícil de definir. Partiendo de su sentido más orgánico, en tanto que líquido en movimiento, el flow en el rap requiere estar provisto de una enorme y cristalina vitalidad en el recitado de los versos, lo que le confiere uno de los aspectos más atractivos al tiempo que complejos en este tipo de música. La autora mencionada, aunque mantiene una ausencia de melodía, nos conduce paulatinamente al significado del concepto que nos ocupa, partiendo de la definición de la música rap:

La musique rap est construite à partir d'échantillons sonores préexistants (samples) - mélodiques ou non-mélodiques, comme des bruits, des crissements, des effets spéciaux, etc. -, assemblés et reconstruits de façon originale par le DJ pour constituer la base sonore sur laquelle le MC débite le texte (Marc, 2007: 3).

Pero realmente ¿qué es el flow? La definición que nos da el Urban dictionary enfatiza en la habilidad del MC (maestro de ceremonias): «La capacidad de un rapero en vocalizar las rimas, en algunos casos complejas, para que encajen entre sí de una manera lógica y sin problemas». Christian Béthune (2003: 235) nos lo define como «la façon dont le MC scande ses rimes, le flow réunit les qualités d'articulation, d'inflexion et de débit propres à chaque rappeur» (Béthune (2003: 13).

Otros autores como Alain Milon se plantean si realmente el rapero canta y, si lo hace, se cuestiona sobre qué tipo de fraseo es o, cómo se explica la excesiva utilización de síncopas y la fluidez del recitado:

Quand on observe le travail scénique du rappeur, sa gestuelle ou son flux verbal, on se rend compte qu'il se porte plus sur l'impact physique que le phrasé produit sur l'auditeur. Le rappeur en imposant un rythme, une force et une violence physique à ses mots frappe les oreilles jusqu'à faire oublier le sens de ses propos. être le haut-parleur pour le rappeur, c’est aussi porter haut et fort la parole de la rue (Milon, 2004: 71)

El investigador Jean-Marie Jacono, de la universidad de Aix en Provence, con el que estamos en contacto, ha desarrollado diferentes estudios que pretenden esclarecer la enorme complejidad del flow, combinando dicción, acentuación de determinadas sílabas o grupos fónicos que generarán un aspecto más amplio con la declamación de las rimas. Nos explica que es imposible no hacer referencia a la declamación (entendida como este concepto complejo y fluido) en los textos de 
Abd al Malik, cita: «Le rap est aussi un art, un art de la déclamation, tout d'abord». Procedente de la locución americana to rap, es decir, entre chismorrear o darle al pico o, simplemente contar cualquier cosa, el rap song puede definirse como la dicción medio cantada y medio hablada de textos elaborados, rimados y ritmados sobre una base musical de mezclas de extractos de otras fuentes sonoras denominadas samples. George Lapassade y Philippe Rouselot (1998: 11), señalan el nacimiento del rap en Nueva York en la década de los 70. La población de origen negro americana demandaba un género que les ayudara a evadirse y que encontraban en la música jamaicana.

\section{Contexto sociocultural del rap en Francia}

El rap en Francia constituye hoy en día una de las señas de identidad profundamente marcadas entre jóvenes de diferentes creencias y no necesariamente de un estrato social determinado. Bien es verdad que algunos barrios parisinos como Barbés o las periferias o banlieues de diferentes ciudades como Marsella han desarrollado de forma importante este género u otros como el raï. Pensemos que es la época de la cassette, formato que contribuyó a la rápida y fácil difusión de estos estilos gracias a su sencilla reproducción. Es necesario mencionar además que el rap forma parte de la cultura o movimiento postmoderno del hip hop, unido al grafiti y a otras estéticas y manifestaciones urbanas. Richard Shusterman en su Estética Pragmatista de 2002 realiza en un alegato reivindicando el rap dentro de esta tendencia: «El rap no solo ejemplifica notablemente las características postmodernas sino que las destaca y tematiza conscientemente (Shusterman, 2002: 269).»

\section{Abd al Malik: biografía}

Aunque nacido en Francia, Abd al Malik, cuyo nombre real es Régis Fayette-Mikano, procede de una familia acomodada congoleña. Tras un abandono inesperado e injustificado de su padre, la madre de Régis, junto con sus hermanos se instala en un HLM de una barriada difícil en Estrasburgo. Coqueteos con la venta de drogas, pequeños robos y amistades no del todo recomendables conviven de forma casi inexplicable con una muy correcta incluso brillante realización de los estudios primarios. Una institutriz, la Srta. Schaeffer que tuvo en clase de maternelle y advirtió su gran capacidad, realiza diferentes gestiones para que pudiera estudiar en el centro privado católico Sainte-Anne, cercano al barrio periférico de Neuhof (Abd al Malik, 2014: 22). Según nos corroboraron sus profesoras de varias materias en un grupo de discusión que llevamos a cabo en el mencionado centro educativo, Régis era un alumno brillante aunque no excesivamente trabajador. Una de ellas, Maryvonne Gelu, recordaba conmovida, prácticamente de memoria, fragmentos de sus redacciones:

il était relativement assidu, mais c’est un enfant que je n’avais jamais noté, évalué, il travaillait à l'oral chez moi, et était très brillant. J'avais noté qu'il ne travaillait pas trop, quand il fallait travailler, il venait librement et volontairement, il n'était pas noté donc il avait une espèce de liberté et très brillant à l’oral (Gelu, 2015).

Abd al Malik ha dicho en varias ocasiones que uno de los profesores que más le marcó fue el Sr. Miry, al que también entrevistamos en su actual instituto católico Notre-Dame. Nos comentaba alguna de sus intervenciones en clase de filosofía, cuestionando de forma incisiva sus explicaciones. Recapitulamos y vemos cómo aquel niño congoleño abandonado por su padre y residente en un HLM consigue, posteriormente, una doble titulación en filosofía y letras clásicas por la Universidad de Estrasburgo.

Innumerables han sido sus intervenciones en televisión en diferentes contextos que van desde su pertenencia al grupo New African Poets (muchos medios le acreditan su creación aunque no fue así) hasta su trayectoria en solitario o acompañado de su mujer la también cantante Wallen. No entramos a valorar si esta excesiva mediatización podría haberle causado cierto desgaste.

Desde el punto de vista de sus creencias religiosas, muy presentes en su obra y en sus canciones, se produce un inhabitual peregrinaje desde una primera etapa de pertenencia al catolicismo (sus padres también lo eran), y su conversión al Islam con dieciséis años, haciéndose llamar Abd al Malik. Como él mismo afirma: «ma quête spirituelle n'est pas assouvie et pour trouver une réponse je m’intéresse aux grands mystiques de l’Islam, je lis les ouvrages d’Ibn Arabi, de Rumi, d’Abd 
el-Kader.» Es por ello que adapta su creencia hacia el sufismo. En 1999 realiza un viaje en compañía de Fabien Coste, su productor, a Marruecos para conocer al maestro sufí Sidi Hamza al-Qâdiri al-Butchichi. Este líder espiritual se encuentra entre los poquísimos denominados «maîtres du soufre rouge» para significar su rareza. «J’ai le sentiment d’avoir enfin trouvé le chemin (Paris Match, 2012).» Precisamente en el capítulo Vers l'universel de su novela autobiográfica de 2004 Qu'Allah bénisse la France, llevada posteriormente al cine en 2014, nos explica que en esta visita, al estar en su presencia, sufrieron una especie de catarsis y fueron sumidos en un estado de amor. Siegfried Foster (2014) señalaba acertadamente, con motivo de la adaptación cinematográfica de la novela mencionada, su recorrido: «du slam à l’islam et du rap à la réalisation, tel est le chemin en zigzag pris par Abd al Malik». Diez años después de haber escrito su novela, el film revela también que fue precisamente el Islam quien le permitió amar a la République.

\title{
3. El flow en Abd al Malik
}

El propósito de esta aportación es centrarnos en el rap de Abd al Malik, nuestro autor, y su particular flow sumando un valor añadido con esa vertiginosa, fluida, chispeante y clara escansión en sus rimas. Pese a que no podríamos asegurar que exista armonía, entendida como el aspecto técnico que nos permitiría establecer los puntos de tensión y distensión, podríamos realizar un estudio a partir de los puntos vocales donde aparece un acento. Así pues, la observación y determinación de la acentuación en el rap del autor, nos lleva a establecer categorías basadas en los puntos en los que el artista incide o enfatiza su dicción.

Toute déclamation dans le rap est fondée sur des accents qui peuvent mettre en valeur les accents de la langue ou les reconfigurer. Le rappeur peut également choisir de les placer sur les deuxième et quatrième temps de la mesure généralement utilisée dans le rap (4/4), qui sont marqués par des samples de batterie (Jocono, 2004: 48).

Hemos seleccionado unos temas de diferentes etapas creativas por mostrar tanto su evolución como la diferente concepción del flow. Empezaremos con un tema de su primera etapa en New African Poets. Aunque muchos medios otorgan la creación a Régis de este grupo, la verdad es que no fue él sino que fueron su hermano Arnaud (conocido por Bilal) y su primo Aïssa. En 1994, consiguen publicar su primer maxi, Trop beau pour être vrai y en 1996 su primer album, La racaille sort un disque, del que hemos elegido el tema Je viens des quartiers para presentar en esta comunicación. Podemos observar aquí aspectos del contexto social que representan un motivo recurrente en sus canciones. El barriogueto constituido por los HLM de la banlieue de Neuhof de Estrasbourgo, casi en su totalidad habitado por negros y magrebíes, de la tercera generación de inmigración a Francia. Se erige pues como foco de creación artística tanto musical como plástica. Música rap, raï, grafiti, hip hop, break dance, adquieren importancia y crean un espacio cultural propio y activo. El fragmento nos revela lo qué significa para esta juventud el barrio:

\author{
«Tu vois mec, c'est comme ça [...] moi je viens des quartiers et je ne l'oublierai jamais» \\ Maintenant tu vois ce que pense quelqu'un comme moi \\ Aucune opportunité, mon quartier est mon étau \\ Mon complexe est gros, ce n'est pas une info \\ Mais qu'est-ce qu'est urgent, à part ton argent \\ Voler est ici un trampoline \\ Le négro le plus nègre a vécu en HLM \\ Où le business circule comme le sang dans les veines
}

Vemos en estos versos, además de una descripción de la Téci ou la Cité, un auto encasillamiento como lascar o racaille, denominándose así por ellos mismos. No niegan de dónde proceden sino que de alguna manera están justificando que su situación les viene dada por las circunstancias de pertenencia a ese gueto que encontramos en el estribillo de este tema: «Tu vois mec, c’est comme ça [...] moi je viens des quartiers et je ne l’oublierai jamais». Aun así, el desánimo, la carencia de objetivos, cierta apología del robo, la vivienda HLM que los estigmatiza, tráfico de drogas, no parecen ser óbice para el desarrollo de la creatividad en esta primera fase de Abd al Malik. Él mismo manifiesta en 2013, en un programa de 
máxima audiencia dirigido por Michael Drucker, que a los 11 años ya había leído a Albert Camus. Respecto al flow de este primer fragmento podemos decir que se trata de un ritmo ciertamente pausado y que mantiene una cadencia de marcada estabilidad. A ello añadimos la alternancia de diferentes intervenciones comenzando por Abd al Malik, Aïssa, (su primo) Bilal (su hermano Arnaud) y los otros miembros de NAP, Karim, Mohammed y Mustapha.

Nos centramos a continuación en Le face à face de coeurs de 2004, su primer álbum como solista. Podemos afirmar que la exégesis del mismo está determinada por la aceptación del sufismo a partir de la influencia ejercida en él por el mencionado maestro Sidi Hamza. En una entrevista para soufisme.org en 2005, al ser preguntado por el título del álbum hace alusión al libro homónimo de Faouzi Skali, Le face à face des cœurs: le soufisme aujourd'hui, publicado por Éditions du Relié, de 2002, al que califica como una de las figuras que ha marcado su recorrido espiritual (Al Malik, 2014: 181). Al parecer, Abd al Malik descubre a este autor por uno de los miembros fundadores de NAP, Mohammad, que, como él, participó en una profunda búsqueda espiritual. Le propone la lectura de Traces de lumière, también de Skali: «ce petit livre solaire devait m’illuminer comme aucun autre auparavant [...] j’étais enthousiasmé par cette littérature soufie qui me bouleversait parfois au point de me faire pleurer» (Al Malik, 2014 :149). El capítulo En chemin vers l'Autre de su libro Qu'Allah bénisse la France comienza precisamente con los primeros versos que utiliza para su tema Ode à l'Amour: «Lors d'un soir d'ivresse spirituelle à la zawiya, au Maroc, Fabien et moi, rédigeâmes une 'Ode à l'Amour' que je décidai de faire précéder des quelques vers d’Ibn Arabi» (Al Malik, 2014: 179). De esta Ode à l'amour del album Le face à face, hemos seleccionado algunos fragmentos para su análisis y presentación aquí.

1. Il y eut temps où je faisais reproche à mon prochain

2. Si sa vie n'était pas proche de la mienne

3. Mais à présent mon cœur accueille toute forme

4. Il est une prairie pour les gazelles

5. Un cloître pour les moines

6. Un temple pour les idoles

7. Une Kaaba pour le pèlerin

8. Les tables de la Thora et le livre du Coran

9. Je professe la religion de l'amour et quelle que soit

10. La direction que prenne sa monture, cette religion est ma religion et ma foi

11. J'ai pu voir qu'le livre de ma vie n'était pas seulement composé d'encre et de lettres

12. Mon cœur devient blanc comme neige

13. Lorsque je goûte les saveurs du je t'aime

14. Dans ton jardin les fleurs sont multiples mais l'eau est unique

15. Laisse-moi me vêtir de ton amour comme d'une tunique

16. Laisse-moi égrainer le chapelet de mon cœur dans ton souvenir

17. Laisse-moi crier au monde le parfum de mon désir

18. Le ciment de la providence nous lie comme les briques du secret

19. J'étais cuivre tu m'as rendu or toi l'Alchimiste de mon cœur

20. Toi qui a su gommer mes erreurs

21. Tu m'as tendu la main un jour et depuis je suis riche

22. En vérité qui est le pauvre, qui est le riche?

23. Je partirais paré des joyaux que tu m’as remis

24. N'est-ce-pas toi Sidi qui m’a rendu vivant dans cette vie (bis)

Entre los versos 1 al 10 encontramos la cita de Ibn Arabi de Murcia que mencionaba Al Malik. No hemos encontrado una traducción unánime para los tres primeros aunque muestran la misma idea. Por ejemplo, Ignaz Goldziher (2005: 142) traduce como «Il fut un temps où je blâmais mon prochain si sa religion n'était pas proche de la mienne.» Este mismo autor incide en los versos de Arabi y también cita a Djelal al-din: «c'est une erreur absolue de tourner autour de la Ka’ba; si la Ka’ba est privée de son parfum elle est une synagogue. Et si nous sentons dans la synagogue la parfum de l'union avec lui, elle est notre Ka’ba ». Observamos una alusión a diferentes creencias que pueden diferir pero que mantienen en común la búsqueda del amor. 
El primer verso realmente nuevo lo encontramos a partir de: «J’ai pu voir qu’le livre de ma vie n’était pas seulement composé d'encre et de lettres» (verso 11) y finaliza con: «n'est-ce pas toi Sidi qui m’a rendu vivant dans cette vie» (verso 24). Podríamos definir esta letra como una especie de oración dadas las diferentes repeticiones con la misma estructura: «laisse-moi me vêtir, laisse-moi égrainer, laisse-moi crier au monde» (versos, 15, 16, 17), en forma anafórica o, «j’étais cuivre et tu m'as rendu or toi l'Alchimiste de mon cœur» (verso 19) utilizando aquí la hermosa metáfora de la purificación y la sublimación alquímica a través de la unión de los contrarios. Desde nuestro punto de vista, podrían ser consideradas como una letanía recurrente de agradecimiento a Sidi Hamza, que acaba con una alusión o invocación directa a él cerrando este fragmento (verso 24). Con motivo de la presentación de su último trabajo Scarifications de 2015 en los estudios Figaro, que constituye su quinto álbum en solitario, sorprende con la interpretación a capella de este tema, por lo tanto deducimos de gran importancia personal para él.

De Gibraltar, su segundo álbum en solitario de 2006, realizaremos brevemente el análisis del tema principal que lleva el mismo nombre. Encontramos un pasaje sin palabras, que corresponde al Estrecho. Es necesario volver a releer varias veces la canción para comprender la historia del joven marroquí que sale de su terrible situación en busca de sustento y felicidad hacia Europa.

Tabla 1. Gibraltar

Sur le détroit de Gibraltar, y’a un jeune noir qui pleure un rêve qui prendra vie, une fois passé Gibraltar.

Sur le détroit de Gibraltar, y’a un jeune noir qui se d'mande si l'histoire le retiendra comme celui qui portait le nom de cette montagne.

Sur le détroit de Gibraltar, y’a un jeune noir qui meurt sa vie bête de "gangsta rappeur" mais ...

Sur le détroit de Gibraltar, y’a un jeune homme qui va naître, qui va être celui qu'les tours empêchaient d'être.

Sur le détroit de Gibraltar, y’a un jeune noir qui boit, dans ce bar où les espoirs se bousculent, une simple canette de Fanta.

Il cherche comme un chien sans collier le foyer qu'il n'a en fait jamais eu, et se dit que p't-être, bientôt, il ne cherchera plus.

Et ça rit autour de lui, et ça pleure au fond de lui.

Faut rien dire et tout est dit, et soudain ... soudain il s'fait derviche tourneur,

Il danse sur le bar, il danse, il n'a plus peur, enfin il hurle comme un fakir, de la vie devient disciple.

Sur le détroit de Gibraltar y’a un jeune noir qui prend vie, qui chante, dit enfin « je t'aime » à cette vie.

Puis les autres le sentent, le suivent, ils veulent être or puisqu'ils sont cuivre.

Comme ce soleil qui danse, ils veulent se gorger d'étoiles, et déchirer à leur tour cette peur qui les voile.
Sur le détroit de Gibraltar, y’a un jeune noir qui n'est plus esclave, qui crie comme les braves, même la mort n'est plus entrave.

Il appelle au courage celles et ceux qui n'ont plus confiance, il dit : "ramons tous à la même cadence!

Dans le bar, y'a un pianiste et le piano est sur les genoux, le jeune noir tape des mains, hurle comme un fou.

Fallait qu'elle sorte cette haine sourde qui le tenait en laisse, qui le démontait pièce par pièce.

Sur le détroit de Gibraltar, y’a un jeune noir qui enfin voit la lune le pointer du doigt et le soleil le prendre dans ses bras. Maintenant il pleure de joie, souffle et se rassoit.

Désormais l'Amour seul, sur lui a des droits. Sur le détroit de Gibraltar, un jeune noir prend ses valises, sort du piano bar et change ses quelques devises,

Encore gros d'émotion il regarde derrière lui et embarque sur le bateau.

Il n’est pas réellement tard, le soleil est encore haut.

Du détroit de Gibraltar, un jeune noir vogue, vogue vers le Maroc tout proche.

Vogue vers ce Maroc qui fera de lui un homme ...

Sur le détroit de Gibraltar ... sur le détroit de Gibraltar

Vogue, vogue vers le merveilleux royaume du Maroc,

Sur le détroit de Gibraltar, vogue, vogue vers le merveilleux royaume du Maroc ... 
Leyendo desde el principio el texto correspondiente a esta composición, se podría pensar en la lucha y el sufrimiento de la inmigración africana que intenta llegar a través de las ciudades españolas de Ceuta y Melilla, o cruzando el estrecho. Pero, casi llegando al final, Abd nos sorprende con un giro totalmente inesperado y otra posible interpretación del fragmento cuando nos dice: «Du détroit de Gibraltar, un jeune noir vogue, vogue vers le Maroc tout proche.» Quizá nos esté dando a entender que se produce con este joven el camino contrario, opuesto, por la decepción que le ha causado poder pertenecer a aquéllos que impiden sistemáticamente el paso hacia un mundo mejor al que todos deberíamos tener derecho. Aprovecharemos también la selección de este tema para indicar una de las características inherentes al rap: la utilización de samples o préstamos tomados de otras canciones o artistas, siendo en este caso el obstinato musical del tema Sinnerman de Nina Simone.

Abd al Malik, como estamos comprobando, no es un rapero cualquiera. Otra de sus características destacadas, que proporcionan un toque verdaderamente sorprendente y llamativo a sus canciones, es la utilización de, o bien fragmentos compuestos ex profeso para orquesta sinfónica (como es el caso de su conocida y emotiva C'est du lourd) o bien la utilización del piano con una función protagonista, no solo de acompañamiento. Un piano que adquiere pues una finalidad diegética formando parte de la misma narración. Para ello, Abd se ha rodeado de grandes maestros como Gérard Jouannest, creador del famoso tema Ne me quitte pas, que logró componer a partir de una melodía que Jacques Brel tenía en su cabeza y que no lograba desarrollar. Jouannest establece el nexo y propicia la admiración de Abd por Jacques Brel. De hecho en el ensayo sobre Brel titulado L'éternel adolescent de Serge Le Vaillant (2008) encontramos un prólogo de Jouannest y otro de Abd al Malik. Jouannest ha compuesto, además del C'est du lourd mencionado temas como La Gravité, Il se rêve debout y L'Alchimiste, pertenecientes al álbum Gibraltar. Él mismo propicia algunas actuaciones de Abd con su esposa Juliette Greco.

\section{Conclusión}

Queremos cerrar ya este breve recorrido y la aproximación al particular flow de nuestro rapero con la colaboración en sus producciones del gurú del tecno Laurent Garnier. Junto a él realiza la banda sonora de la adaptación cinematográfica de su novela Qu'Allah bénisse la France. Realmente, desde nuestro punto de vista, la intervención de Laurent Garnier ha supuesto un verdadero giro en su carrera musical, otorgando a los temas una viveza y un nuevo tratamiento del flow que destaca por una textura musical basada en capas arpegiadas de diferentes acordes muy luminosos, lo que otorga una nueva vida a los recitados de Abd al Malik. Hace tan solo unos días la revista musical les Inrocks, con motivo de la presentación de este último álbum comentaba: «Si l'alliance du rap et de la techno étonne, cette collusion supersonique ne doit pourtant rien au hasard. Le rap et la techno sont nés à la même époque, dans les mêmes lieux, derrière les mêmes platines.»

\section{Referencias bibliográficas}

Al MaLiK, Abd (2014). Qu’allah bénisse la france!(troisième ed.). Paris: Albin Michel.

BÉThune, Christian (2004). Pour une esthétique du rap. Paris: Klincksieck.

CuAz, Odile (2012). L'été où... j'ai rencontré mon maître soufi par Abd al Malik. < http://www.parismatch.com/Culture/Cinema/L-eteou-j-ai-rencontre-mon-maitre-soufi-par-Abd-al-Malik-156806 > [Consulta: 10 de abril de 2016] [personal].

FOSTER, Siegfried (2014). «Qu’Allah bénisse la France, le rappeur Abd Al Malik se réalise». Radio France Info, Afrique, 2014 [En ligne] <http://www.rfi.fr/afrique/20141210-allah-benisse-france-rappeur-abd-al-malik-realise-film> [Consulta: 10 de abril 2016].

GELU, Maryvonne (2015). Groupe de discussion. [Consulta: 19 de marzo de 2015] [personal].

GolDzIHER, Ignaz (2005). Le dogme et la loi dans l'islam: histoire du développement dogmatique et juridique de la religion musulmane. Paris: Éditions de l’éclat.

JACONO, Jean-Marie (2004). «Ce que révèle l'analyse musicale du rap: l’exemple de “Je danse le mia” d’IAM». Volume, 3(2), pp. 43-53. 
LAPASSADE, George \& Rousselot, Philippe. (1998). Le rap ou la fureur de dire: Essai (sixième ed.). Paris: Éditions Loris Talmart.

LE VAILlant, Serge (2008). L'éternel adolescent. Paris: Éditions Textuel.

LES INROCKs. Abd al Malik revient avec la rage au ventre sur «Scarifications». <http://www.lesinrocks.com/musique/critiquealbum/abd-al-malik-scarifications/> [Consulta: 12 de abril de 2016] [personal].

MARC, Isabelle (2007). «La voix rap», Littemu [En ligne], Rencontres Sainte-Cécile, 2007, Contributions, mis à jour le: 25/06/2008,

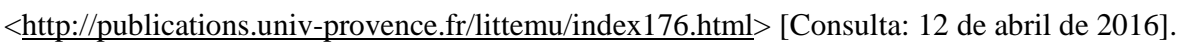

Milon, Alain (2004). «Pourquoi le rappeur chante ? Le rapcomme expression de la relégation urbaine», en Cités3 (n 19) p. 71-80. $<$ http://www.cairn.info/revue-cites-2004-3-page-71.htm> [Consulta: 12 de abril de 2016].

PERrIER, Jean-Claude. (2010). Le rap français: dix ans après; anthologie. Paris: Éditions de la Table Ronde, coll. «La petite Vermillon».

SHUSTERMAN, Richard. (2002). Estética pragmatista: viviendo la belleza, repensando el arte. Barcelona: Idea Books, coll. «Idea Universitaria».

SOUfISME.ORg. Un rap d'amour à écouter avec le coeur. Interview de Abd al Malik chanteur du groupe NAP. $<$ http://www.soufisme.org/2.0/artsculture/litterature/rap-damour-a-ecouter-coeur/> [Consulta: 17 de abril de 2016] [personal]

URBAN DiCTIONARY. Flow. <http://www.urbandictionary.com/define.php?term=Flow> [Consulta: 17 de abril de 2016] 